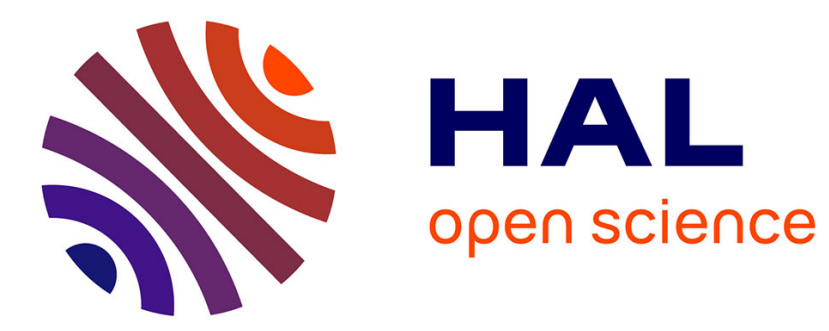

\title{
Chiral Optical Stern-Gerlach Newtonian Experiment
}

Nina Kravets, Artur Aleksanyan, Etienne Brasselet

\section{To cite this version:}

Nina Kravets, Artur Aleksanyan, Etienne Brasselet. Chiral Optical Stern-Gerlach Newtonian Experiment. Physical Review Letters, 2019, 122 (2), pp.024301 (1-). 10.1103/PhysRevLett.122.024301 . hal-02104053

\section{HAL Id: hal-02104053 \\ https://hal.science/hal-02104053}

Submitted on 19 Apr 2019

HAL is a multi-disciplinary open access archive for the deposit and dissemination of scientific research documents, whether they are published or not. The documents may come from teaching and research institutions in France or abroad, or from public or private research centers.
L'archive ouverte pluridisciplinaire HAL, est destinée au dépôt et à la diffusion de documents scientifiques de niveau recherche, publiés ou non, émanant des établissements d'enseignement et de recherche français ou étrangers, des laboratoires publics ou privés. 


\title{
Chiral Optical Stern-Gerlach Newtonian Experiment
}

\author{
Nina Kravets, Artur Aleksanyan, and Etienne Brasselet \\ Université de Bordeaux, CNRS, Laboratoire Ondes et Matière d'Aquitaine, F-33400 Talence, France
}

(Received 28 August 2018; revised manuscript received 6 November 2018; published 16 January 2019)

\begin{abstract}
We report on a chiral optical Stern-Gerlach experiment where chiral liquid crystal microspheres are selectively displaced by means of optical forces arising from optical helicity gradients. The present Newtonian experimental demonstration of an effect predicted at molecular scale [New J. Phys. 16, 013020 (2014)] is a first instrumental step in an area restricted so far to theoretical discussions. Extending the SternGerlach experiment legacy to chiral light-matter interactions should foster further studies, for instance towards the elaboration of chirality-enabled quantum technologies or spin-based optoelectronics.
\end{abstract}

DOI: 10.1103/PhysRevLett.122.024301

The century-old Stern-Gerlach experiment [1] is not only one of the few canonical quantum physics experiments but also a paradigm when dealing with state-selective deflections of particles in a field gradient. In particular, optical analogs of Stern-Gerlach experiments emerged after the advent of the laser [2] and were further enriched by versatile light-matter interaction processes [3-5]. In the multidisciplinary context of sorting chiral entities with opposite handedness, contactless optical Stern-Gerlach approaches have been proposed [6-9], however, without experimental realization to date. Chirality refers to mirror-image entities (enantiomers) that cannot be superimposed by spatial rotations, which dates back almost two centuries ago with the pioneering works of several scientists. In particular, Louis Pasteur, who performed the first chiral resolutionthe process of separating a pair enantiomers - of racemic acid using handmade tweezers to sort crystallites of opposite handedness [10]. Since then, the concept of chirality does not only hold a special place in chemistry but spreads across scientific disciplines like biology, mathematics, and physics.

Nowadays, the need for enantiomers separation is a cornerstone of pharmaceutical and food-processing industries as well as a key ingredient for carbon- and DNA-based technologies [11]. In this context, chiral light emerged as a novel tool to achieve contactless chiral resolution as theoretically discussed by several groups [6-9,12-23]. While some of the proposed approaches have been experimentally addressed at the microscale [24-28], others remain elusive, such as those relying on chirality-selective lateral forces. This is the case of an intriguing chiral optical analog of the Stern-Gerlach experiment for which a chiral entity is selectively displaced along a one-dimensional (1D) gradient of optical helicity [9]. Here we report on the experimental proof of concept of an optical Stern-Gerlach chiral sorter operated in the classical regime, where the particle's deflection is described by Newtonian mechanics.

In our experiments, a light field with a $1 \mathrm{D}$ gradient of helicity density is prepared according to Ref. [29], by interfering two slightly noncollinear laser beams having orthogonal linear polarization states. This is made by using an optical element called a "wedge depolarizer," which consists of a quartz wedge having its optical axis oriented along the wedge slope that defines the $x$ axis, cemented to a fused silica wedge to compensate for the overall deviation of the light flow. Indeed, by illuminating the depolarizer by a normally incident (along the $z$ axis) linearly polarized continuous-wave Gaussian beam at $\lambda_{0}=532 \mathrm{~nm}$ wavelength with a polarization azimuth oriented at $45^{\circ}$ from the $x$ axis, the collinear ordinary and extraordinary waves propagating along the $z$ axis in the quartz wedge are refracted at the quartz-silica interface into two noncollinear beams making an angle $\alpha \sim 2 \mathrm{mrad}$ one with another. Such a single-mirror approach has the advantage to be resilient to mechanical vibrations, in contrast to a conventional twomirror interference scheme. By imaging the plane of the depolarizer with a microscope objective, we thus produce a spatially oscillating helicity density along the $x$ axis with a spatial period $\Lambda=\lambda_{0} / \alpha \simeq 200 \mu \mathrm{m}$ in the plane of the sample. Moreover, in order to ensure practically that the chiral micro-objects with characteristic size $R$ are confined to motion along the direction of the helicity gradients, we use a Gaussian light field having an optical intensity spatial distribution of the form $I(x, y)=I_{0} \exp \left[-2\left(x^{2} / w_{x}^{2}+\right.\right.$ $\left.\left.y^{2} / w_{y}^{2}\right)\right]$ where $I_{0}=2 P /\left(\pi w_{x} w_{y}\right)$ with $P$ is the total optical power, with $R \sim w_{y} \ll w_{x}$. This is made by placing the depolarizer in the focal plane of a cylindrical lens. We refer to the Supplemental Material [30] for a detailed version of the setup depicted in Fig. 1(a).

The assessment of the helicity density $h$ is made accounting that $\alpha \ll 1$ and our paraxial framework. In that case, one can consider that $h(x, y) \propto S_{3}(x, y)=\tilde{h}(x, y)$ where $S_{3}=$ $I_{L}-I_{R}$ is the third Stokes parameter with $I_{L, R}$ being the intensity distributions of the left-handed and right-handed circularly polarized component of the spatially modulated optical field in the plane of the sample [29]. Measurement of 


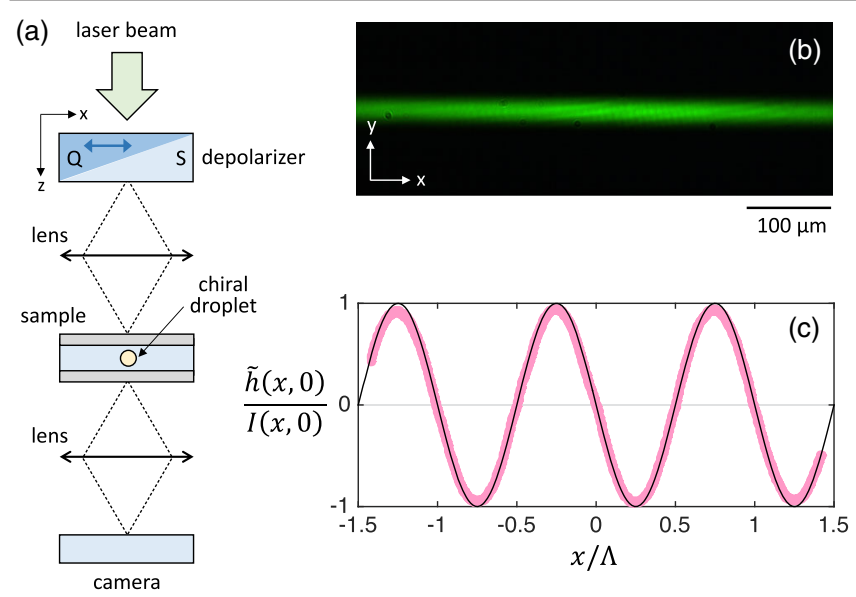

FIG. 1. (a) Sketch of the setup. $Q$ and $S$, respectively, refer to cemented quartz crystal (double arrow shows the optical axis orientation) and silica wedges. The incident laser beam is linearly polarized at $45^{\circ}$ from the $x$ axis. (b) Optical intensity distribution in the plane of the sample having an elliptical Gaussian shape associated with waists radii at $\exp (-2)$ of the maximal intensity $w_{x}=700 \mu \mathrm{m}$ and $w_{y}=20 \mu \mathrm{m}$ along the $x$ and $y$ axes. (c) 1D reduced harmonic optical helicity $\tilde{h}(x, 0) / I(x, 0)$ in the plane of the sample, with spatial period $\Lambda \simeq 200 \mu \mathrm{m}$. Thick red curve: Experimental data obtained by spatial averaging along the $y$ axis in the range $-w_{y} / 2<y<w_{y} / 2$. Thin dark curve: Expected sinusoidal behavior.

the 1D modulation of the helicity along the $x$ axis is shown in Fig. 1(c) (thick curve), which reveals a behavior of the form $\tilde{h}(x, 0)=I_{0} \sin (2 \pi x / \Lambda) \exp \left(-2 x^{2} / w_{x}^{2}\right)$ (thin curve), noting that the depolarizer is appropriately positioned along the $x$ axis to ensure a maximal helicity gradient at the centroid location of the driving light beam that defines the origin $(x, y)=(0,0)$.

We use chiral droplets made of both right-handed $(\chi=+1)$ and left-handed $(\chi=-1)$ helix-based liquid crystal commercial mixtures (BEAM Co.) characterized by a helical pitch $p$. The droplets are prepared by dispersing the liquid crystal into a $40 \mathrm{wt} \%$ aqueous glycerol solution doped with $\sim 1$ wt $\%$ polyvinyl alcohol. This leads to the spontaneous formation of spherical droplets having radial distribution of the helix axis. The resulting onionlike structure of the droplets is depicted in Fig. 2(a). The prepared fluidic mixture is then inserted into a cell made of two glass substrates separated by a $\sim 200 \mu \mathrm{m}$ gap and a hydrophilic surface treatment is made to prevent the droplet from wetting the bottom glass substrate. From the optics of chiral anisotropic media, light propagating along the helix axis is fully transmitted when $h \chi=+1$. On the other hand, when $h \chi=-1$, light experiences the circular Bragg reflection phenomenon [32], which is associated with a photonic band gap defined by $p n_{\perp}<\lambda<p n_{\|}, n_{\|}$and $n_{\perp}$ being the refractive indices along and perpendicular to the liquid crystal molecular orientation. This behavior is assessed using the setup sketched in Fig. 2(b). The average

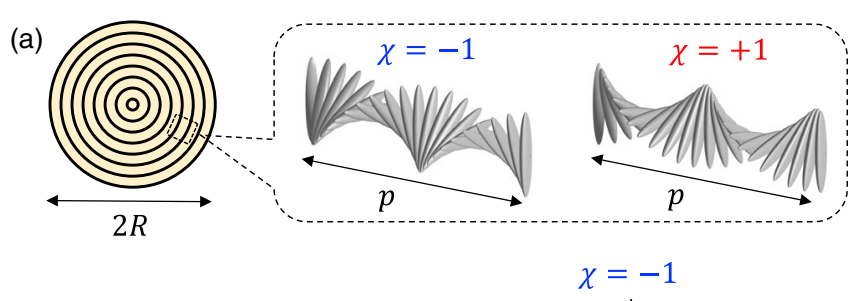

(b)

(c)

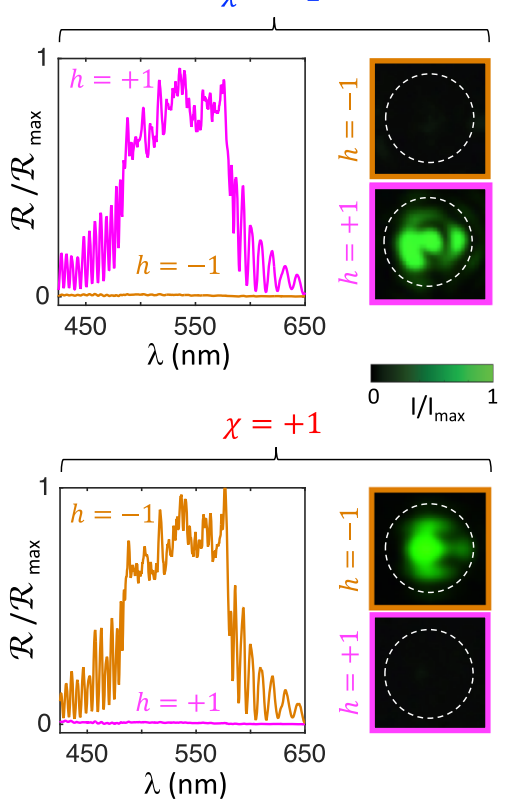

FIG. 2. (a) Onionlike chiral liquid crystal droplet where the layered structure refers to the periodic modulation of the dielectric permittivity tensor of the liquid crystal with the period being half the helical pitch $p$ of the supramolecular helix organization, see Ref. [31] for a detailed structural description. (b) Sketch of the spectroscopic setup. BS: beam splitter; P: linear polarizer; AQWP: achromatic quarter-wave plate. FS: fiber spectrometer. (c) For each of the four combinations $(\chi, h)=( \pm 1, \pm 1)$, the reflection spectrum for a $8 \mu \mathrm{m}$-thick film of the liquid crystal mixture with uniform alignment of helical axis along the normal to the film is shown (left panel) as well as the polarized reflection image, at $532 \mathrm{~nm}$, of a droplet with radius $R \simeq 30 \mu \mathrm{m}$ (right panel, where the contour of the droplet appear as a white dashed circle). Every spectrum is the average of five independent measurements and is normalized by the spectrum obtained from the Fresnel reflection from a bare glass substrate, while $\mathcal{R}_{\max }$ refers to the maximal reflectance of the whole set of measured spectra.

reflection spectra of reference cholesteric films are shown in Fig. 2(c) for the four combinations $(\chi, h)=( \pm 1, \pm 1)$. Prior to recording the light-induced motion of each studied droplet, its handedness is qualitatively identified in situ via its reflection image at $532 \mathrm{~nm}$. This is done by introducing a reflective branch equivalent to Fig. 2(b) to the main setup sketched in Fig. 2(a); typical images are shown in the rightmost part of Fig. 2(c). Since $\lambda_{0}$ falls into the circular photonic band gap, strong chiral-selective light-matter interaction is achieved by design.

Summarizing, the analogy between the original SternGerlach experiment and ours is as follows: the liquid crystal 
(a)

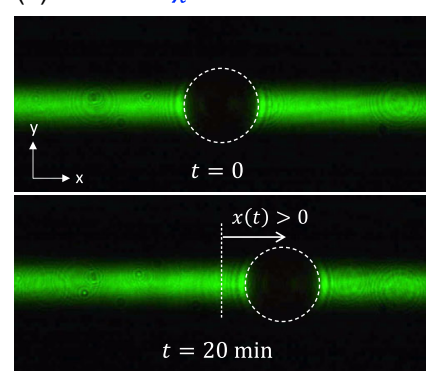

(b)

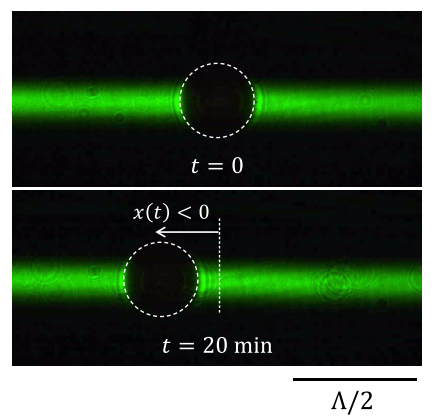

FIG. 3. Direct observation of chiral-selective displacement under an optical helicity gradient illustrated by snapshots of an irradiated droplet with radius $R \simeq 30 \mu \mathrm{m}$ initially placed at $x=0$ at time $t=0$ and after $20 \mathrm{~min}$ illumination by the laser beam with space-varying helicity and total optical power $P=400 \mathrm{~mW}$, for $\chi=-1$ (a) and $\chi=+1$ (b). The dashed white circle refers to the contour of the droplet.

droplets play the role of the silver atoms, the handedness of the droplets plays the role of the spin of the silver atoms, and the optical helicity gradient plays the role of magnetic field gradient. A practical technical difference is that we observe displacements rather than deflection. Note such a framework originality in the context of previous realizations of Stern-Gerlach analogs where light can play either the role of the field gradient [3], that of the deflected particles [4], or even both [5].

Our experimental protocol consists in placing a chiral droplet with radius $R \simeq 30 \mu \mathrm{m}$ at $(x, y)=(0,0)$ and recording its trajectory under constant irradiation. The qualitative demonstration is reported in Fig. 3 where images of the droplet at $t=0$ and after $20 \mathrm{~min}$, for which the steady state is reached, are shown both for left-handed [Fig. 3(a)] and right-handed [Fig. 3(b)] droplets. The observed light-induced transverse displacements along the $x$ axis have opposite sign and final states are located nearby $x= \pm \Lambda / 4$, where the gradient of helicity vanishes [Fig. 1(c)]. On the other hand, quantitative analysis is assessed by retrieving the droplet trajectories from the recorded data. The results are illustrated in Fig. 4(a) which presents the average trajectory and standard deviation associated with measurements made over $\sim 10$ independent events made with different droplets of approximately same radius, both for $\chi= \pm 1$. Chiral-selective transverse deviation under the helicity gradient is therefore clearly demonstrated experimentally. In contrast, no clear discriminatory trend is observed in the control experiment without helicity gradients (this is made by removing the depolarizer), as shown in Fig. 4(b).

The quantitative support is provided from chiral Bragg droplet optomechanics knowledge. Indeed, the vectorial force field can be calculated from a simplified ray-optics model according to Ref. [28]. The latter model is written for axisymmetric beams illuminating a droplet, from which

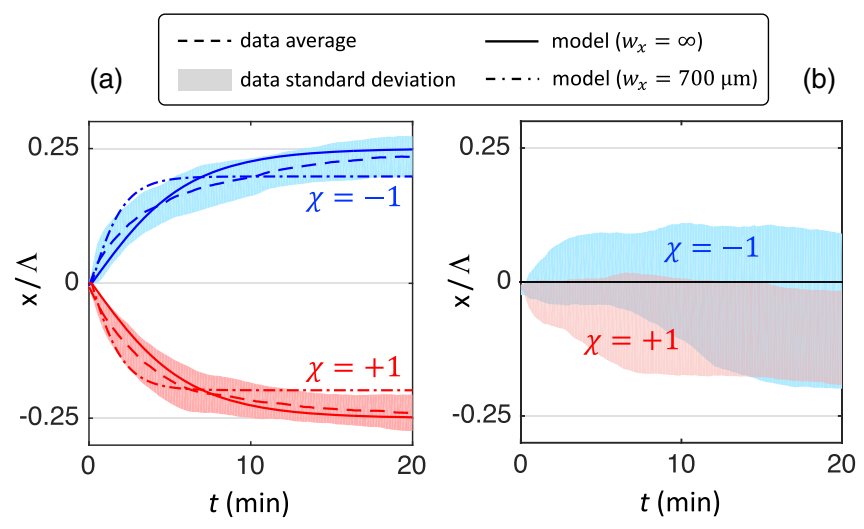

FIG. 4. Quantitative analysis of chiral-selective displacement by discriminatory lateral optical forces illustrated by the displacement dynamics over 20 min duration illumination. (a) Discriminatory dynamics for the same conditions as in Fig. 3. Dashed curves refer to the average over $\sim 10$ independent measurements and colored bands refer to the corresponding standard deviation. Solid lines: Simulations according to the model discussed in the text. Blue color: $\chi=-1$; red color: $\chi=+1$. (b) Control experiment using a uniform linearly polarized with null helicity, all other parameters being the same as in panel (a).

one can derive the expressions for the lateral optical force field in the plane $(x, y), \mathbf{F}=F_{x} \mathbf{x}+F_{y} \mathbf{y}$. In short, the model consists in summing up the elementary contributions of the linear momentum transfer at the surface of the droplet. The droplet is associated with the usual polar and azimuthal angles $(\theta, \phi)$ in the spherical coordinate originating at the droplet center, noting that $\theta=0$ points towards the beam propagation direction (i.e., the $z$ axis). A key parameter is the angle $\vartheta_{B}$ [33] that characterizes the ability of the chiral liquid crystal to reflect light via the circular Bragg reflection phenomenon. Namely, if an incident ray impinges at the surface of the droplet with a local incidence angle lower than $\vartheta_{B}$ the light is considered totally reflected if $h=-\chi$ while all other situations are treated as if the droplet is a dielectric sphere with refractive index $n=\left(n_{\|}+n_{\perp}\right) / 2$ being the mean of the refractive index parallel and perpendicular to the liquid crystal main molecular axis. The result of calculations for $\chi=-1$ is

$$
\begin{aligned}
\mathbf{F}(x, y)= & -\frac{n_{\mathrm{ext}} R^{2}}{2 c}\left(\int_{0}^{2 \pi} \int_{\pi / 2}^{\pi-\vartheta_{B}}\{I(X, Y) f(\theta)\right. \\
& \times[\cos \phi \mathbf{x}+\sin \phi \mathbf{y}] \sin 2 \theta\} d \theta d \phi \\
& +\int_{0}^{2 \pi} \int_{\pi-\vartheta_{B}}^{\pi}\{I(X, Y)[S(X) f(\theta)+C(X) \sin 2 \theta] \\
& \times[\cos \phi \mathbf{x}+\sin \phi \mathbf{y}] \sin 2 \theta\} d \theta d \phi)
\end{aligned}
$$

$n_{\text {ext }}$ is the refractive index of the outer fluid, $c$ is the speed of light, 


$$
\begin{gathered}
X(x, \theta, \phi)=x+R \sin \theta \cos \phi, \\
Y(y, \theta, \phi)=y+R \sin \theta \sin \phi, \\
S(X)=\sin ^{2}(\pi X / \Lambda+\pi / 4), \\
C(X)=\cos ^{2}(\pi X / \Lambda+\pi / 4),
\end{gathered}
$$

and

$$
f(\theta)=\mathcal{R} \sin 2 \theta-\mathcal{T}^{2} \frac{\sin \left(2 \theta-2 \theta_{\text {int }}\right)+\mathcal{R} \sin 2 \theta}{1+2 \mathcal{R} \cos 2 \theta_{\text {int }}+\mathcal{R}^{2}},
$$

where $\mathcal{R}$ and $\mathcal{T}=1-\mathcal{R}$ are the reflectance and transmittance of the droplet interface, with $\theta_{\text {int }}=$ $-\arcsin \left[\left(n_{\text {ext }} / n\right) \sin \theta\right]$ the signed angle of refraction. Accounting for the incident circular polarization state and discarding the polarization projection on the local frame of incidence, we also assume that $\mathcal{R}(\theta)=\left[\mathcal{R}_{\|}(\theta)+\right.$ $\left.\mathcal{R}_{\perp}(\theta)\right] / 2 \quad$ with $\quad \mathcal{R}_{\|}(\theta)=\left[\tan \left(\theta-\theta_{\text {int }}\right) / \tan \left(\theta+\theta_{\text {int }}\right)\right]^{2}$ and $\mathcal{R}_{\perp}(\theta)=\left[\sin \left(\theta-\theta_{\text {int }}\right) / \sin \left(\theta+\theta_{\text {int }}\right)\right]^{2}$ the reflectance coefficients of plane waves polarized parallel and perpendicular to the incidence plane [34]. For $\chi=+1$, one simply needs to make the formal change $S \leftrightarrow C$ in Eq. (1).

The parameters entering in the simulations are chosen according to what follows. Namely, the refractive index of the $\simeq 40 \mathrm{wt} \%$ aqueous glycerol solution doped with $1 \mathrm{wt} \%$ polyvinyl alcohol is taken as $n_{\text {ext }} \simeq 1.38$ from the tabulated data of aqueous glycerol mixture at $20^{\circ} \mathrm{C}$ and $589 \mathrm{~nm}$ wavelength and its dynamic viscosity as $\eta=3.72 \mathrm{mPas}$ [35]. The used optical power is $P=400 \mathrm{~mW}$, and we use $w_{x}=700 \mu \mathrm{m}$ and $w_{y}=20 \mu \mathrm{m}$. The average refractive index $n$ of the liquid crystal mixture is evaluated from the optics of circular Bragg reflection that gives $\Delta \lambda=$ $p\left(n_{\|}-n_{\perp}\right)$ for the circular photonic band gap width. Indeed, we measure $\Delta \lambda \simeq 100 \mathrm{~nm}$ [Fig. 2(c)] and the liquid crystal data sheet gives the birefringence $n_{\|}-n_{\perp}=0.343$ at $532 \mathrm{~nm}$ wavelength and room temperature. This gives $p \simeq 290 \mathrm{~nm}$. The central wavelength of the band gap equals $n p \simeq 530 \mathrm{~nm}$, which gives $n \simeq 1.82$. This leaves the characteristic Bragg angle $\vartheta_{B}$ as the single adjustable parameter of the model.

The dynamics of the droplets is theoretically retrieved from Newtonian mechanics, noting that the Reynolds number associated with the fluid flow around the moving droplet is small with respect to unity. This implies that the drag viscous force $\left(F_{\text {drag }}\right)$ exerted on the droplet in a direction opposite to its displacement is balanced by the optical force $F_{x}$. Since $F_{\text {drag }}=6 \pi \eta R(\partial x / \partial t)$, one gets $x(t)=\int_{0}^{t} F_{x}\left(x\left(t^{\prime}\right), 0\right) /(6 \pi \eta R) d t^{\prime}$. By minimizing the standard deviation between simulations (dash-dotted curve) and experimental data (dashed curve) over the full dynamics, see Fig. 4(a), one obtains $\vartheta_{B} \simeq 19^{\circ}$. The corresponding force field exerted on the droplets in the plane $(x, y)$ is shown in Fig. 5 where the $x$ and $y$ contributions, namely, $F_{x}$ and $F_{y}$, are displayed. The 1D displacement along the $x$ axis irrespective of the material chirality is explained by the fact that $F_{y}$ acts as restoring force towards $y=0$ at all times, both for $\partial h / \partial x \neq 0$ [Fig. 5(a)] and $\partial h / \partial x=0$ [Fig. 5(b)]. In the presence of the helicity gradient, the spatially modulated $F_{x}$ component is maximal at the origin, its sign is that of $\chi(\partial h / \partial x)$ and its magnitude vanishes at $\left|x_{\infty}\right| \lesssim \Lambda / 4$, which corresponds to the asymptotic location of the droplet. The slight offset between $x_{\infty}$ and the location of zero helicity is due to the nonvanishing restoring contribution towards the origin due to the finite value of $w_{x}$. However, one notes that imposing $w_{x} \rightarrow \infty$ gives even better agreement between simulations and observations, see solid curve in Fig. 4(a) for which $\vartheta_{B} \simeq 15^{\circ}$. This is ascribed to the residual intensity modulation along the $x$ axis that practically washes out the intensity gradient force contribution along this axis. Finally, in the absence of a helicity gradient, both $F_{x}$ and $F_{y}$ are null at the origin, which subsequently corresponds to a $2 \mathrm{D}$ optical trapping configuration. Still, uncontrolled random drift of the droplets is observed [Fig. 4(b)]. It is associated with an average maximal velocity much weaker than in the case $\partial h / \partial x \neq 0$. This is ascribed to the fact that residual contributions (residual flows, temperature gradients, mechanical vibrations, spatial noise of the intensity profile, etc.) are at work

(a) Nonzero helicity gradient

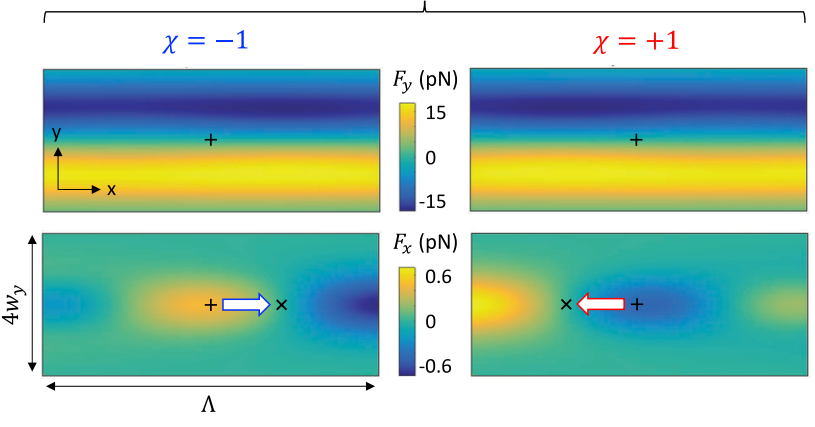

(b) Zero helicity gradient

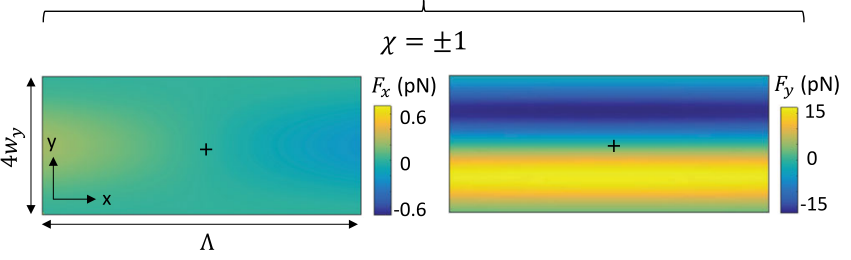

FIG. 5. Calculated maps of the $x$ and $y$ components of the optical force field exerted on the droplet, $F_{x}$ and $F_{y}$, in the plane $(x, y)$ for $\chi= \pm 1$, in the presence (a) and absence (b) of a helicity gradient using all experimental values for all the model parameters and $\vartheta_{B} \simeq 19^{\circ}$ from the adjustment described in the text. Straight cross markers indicate the origin, where the droplet is placed at $t=0$. Oblique cross markers in panel (a) indicate the asymptotic position of the droplet after $20 \mathrm{~min}$. 
when $\partial h / \partial x=0$, while the droplets remain confined to motion along the $x$ axis owing to intensity gradient forces along the $y$ axis. We also note that the slight imbalance (regarding the material handedness) is perhaps due to not perfectly sufficient statistics.

These results invite for further tests of recent theoretical efforts regarding chiral light-matter interaction [36-42]. Since chirality is perceived as a key actor towards answering universal questions such as the prevalence of a given handedness in living systems [43] or implementing nonreciprocal photonics technologies $[44,45]$, present work calls for chiral optomechanics developments, such as chiral resolution, especially at the nanoscale, where quantum effects come to prominence. Finally, noting that the SternGerlach experiment has inspired many branches of physics, for instance astrophysics with the quest for hypothetical elementary particles [46], our work exemplifies that analogy remains a powerful tool worth considering on experimental grounds.

We thank J. Leng for the hydrophilic agent Mirapol Surf S 500 used to treat the bottom glass substrates. This study has been carried out with financial support from the French National Research Agency (ANR) in the frame of the "Investments for the future" Programme IdEx Bordeaux LAPHIA (ANR-10-IDEX-03-02).

*etienne.brasselet@u-bordeaux.fr

[1] W. Gerlach and O. Stern, Der experimentelle nachweis der richtungsquantelung im magnetfeld, Z. Phys. 9, 349 (1922).

[2] A. Kazantsev, Recoil effect in a strong resonant field, $\mathrm{Zh}$. Eksp. Teor. Fiz. 67, 1660 (1974) [Sov. Phys. JETP 40, 825 (1975)].

[3] T. Sleator, T. Pfau, V. Balykin, O. Carnal, and J. Mlynek, Experimental Demonstration of the Optical Stern-Gerlach Effect, Phys. Rev. Lett. 68, 1996 (1992).

[4] L. Karpa and M. Weitz, A Stern-Gerlach experiment for slow light, Nat. Phys. 2, 332 (2006).

[5] A. Karnieli and A. Arie, All-Optical Stern-Gerlach Effect, Phys. Rev. Lett. 120, 053901 (2018).

[6] Y. Li, C. Bruder, and C. Sun, Generalized Stern-Gerlach Effect for Chiral Molecules, Phys. Rev. Lett. 99, 130403 (2007).

[7] B. Spivak and A. Andreev, Photoinduced Separation of Chiral Isomers in a Classical Buffer Gas, Phys. Rev. Lett. 102, 063004 (2009).

[8] X. Li and M. Shapiro, Theory of the optical spatial separation of racemic mixtures of chiral molecules, J. Chem. Phys. 132, 194315 (2010).

[9] R. P. Cameron, S. M. Barnett, and A. M. Yao, Discriminatory optical force for chiral molecules, New J. Phys. 16, 013020 (2014).

[10] L. Pasteur, Recherches sur les relations qui peuvent exister entre la forme cristalline, la composition chimique et le sens de la polarisation rotatoire, Ann. Phys. Chem. 24, 442 (1848).
[11] J. Zhang, M. T. Albelda, Y. Liu, and J. W. Canary, Chiral nanotechnology, Chirality 17, 404 (2005).

[12] A. Canaguier-Durand, J. A. Hutchison, C. Genet, and T. W. Ebbesen, Mechanical separation of chiral dipoles by chiral light, New J. Phys. 15, 123037 (2013).

[13] D. S. Bradshaw and D. L. Andrews, Chiral discrimination in optical trapping and manipulation, New J. Phys. 16, 103021 (2014).

[14] S. B. Wang and C. T. Chan, Lateral optical force on chiral particles near a surface, Nat. Commun. 5, 3307 (2014).

[15] A. Hayat, J. B. Mueller, and F. Capasso, Lateral chiralitysorting optical forces, Proc. Natl. Acad. Sci. U.S.A. 112, 13190 (2015).

[16] M.H. Alizadeh and B. M. Reinhard, Transverse chiral optical forces by chiral surface plasmon polaritons, ACS Photonics 2, 1780 (2015).

[17] A. Canaguier-Durand and C. Genet, Plasmonic lateral forces on chiral spheres, J. Opt. 18, 015007 (2016).

[18] H. Chen, C. Liang, S. Liu, and Z. Lin, Chirality sorting using two-wave-interference-induced lateral optical force, Phys. Rev. A 93, 053833 (2016).

[19] Y. Zhao, A. A. Saleh, and J. A. Dionne, Enantioselective optical trapping of chiral nanoparticles with plasmonic tweezers, ACS Photonics 3, 304 (2016).

[20] T. Zhang, M. R. C. Mahdy, Y. Liu, J. H. Teng, C. T. Lim, Z. Wang, and C.-W. Qiu, All-optical chirality-sensitive sorting via reversible lateral forces in interference fields, ACS Nano 11, 4292 (2017).

[21] P. Acebal, L. Carretero, and S. Blaya, Design of an optical conveyor for selective separation of a mixture of enantiomers, Opt. Express 25, 32290 (2017).

[22] T. Cao and Y. Qiu, Lateral sorting of chiral nanoparticles using fano-enhanced chiral force in visible region, Nanoscale 10, 566 (2018).

[23] G. Pellegrini, M. Finazzi, M. Celebrano, M. A. Iatì, O. M. Maragò, P. Biagioni et al., Superchiral surface waves for alloptical enantiomer separation, arXiv:1803.10010.

[24] R. J. Hernandez, A. Mazzulla, A. Pane, K. VolkeSepulveda, and G. Cipparrone, Attractive-repulsive dynamics on light-responsive chiral microparticles induced by polarized tweezers, Lab Chip 13, 459 (2013).

[25] G. Tkachenko and E. Brasselet, Spin Controlled Optical Radiation Pressure, Phys. Rev. Lett. 111, 033605 (2013).

[26] G. Tkachenko and E. Brasselet, Optofluidic chiral sorting of material chirality by chiral light, Nat. Commun. 5, 3577 (2014).

[27] M. Donato, J. Hernandez, A. Mazzulla, C. Provenzano, R. Saija, R. Sayed, S. Vasi, A. Magazzu, P. Pagliusi, R. Bartolino, P. Gucciardi, O. Marago, and G. Cipparrone, Polarization-dependent optomechanics mediated by chiral microresonators, Nat. Commun. 5, 3656 (2014).

[28] G. Tkachenko and E. Brasselet, Helicity-dependent threedimensional optical trapping of chiral microparticles, Nat. Commun. 5, 4491 (2014).

[29] R. P. Cameron, S. M. Barnett, and A. M. Yao, Optical helicity of interfering waves, J. Mod. Opt. 61, 25 (2014).

[30] See Supplemental Material at http://link.aps.org/ supplemental/10.1103/PhysRevLett.122.024301 for a detailed version of the setup depicted in Fig. 1(a). 
[31] D. Sec, T. Porenta, M. Ravnik, and S. Zumer, Geometrical frustration of chiral ordering in cholesteric droplets, Soft Matter 8, 11982 (2012).

[32] M. Faryad and A. Lakhtakia, The circular bragg phenomenon, Adv. Opt. Photonics 6, 225 (2014).

[33] Note that the angle $\vartheta_{B}$ refers to the angle labeled $\theta_{\text {ext }, B}$ in Ref. [28].

[34] M. Born and E. Wolf, Principles of Optics (Pergamon, New York, 2005).

[35] Glycerine Producers' Association, Physical Properties of Glycerine and Its Solutions (Glycerine Producers' Association, New York, 1963), p. 13.

[36] M. Nieto-Vesperinas, J. J. Saenz, R. Gomez-Medina, and L. Chantada, Optical forces on small magnetodielectric particles, Opt. Express 18, 11428 (2010).

[37] K. Y. Bliokh, Y. S. Kivshar, and F. Nori, Magnetoelectric Effects in Local Light-Matter Interactions, Phys. Rev. Lett. 113, 033601 (2014).

[38] A. Canaguier-Durand and C. Genet, Chiral route to pulling optical forces and left-handed optical torques, Phys. Rev. A 92, 043823 (2015).

[39] I. Fernandez-Corbaton, M. Fruhnert, and C. Rockstuhl, Objects of Maximum Electromagnetic Chirality, Phys. Rev. X 6, 031013 (2016).
[40] R. P. Cameron, J. B. Götte, S. M. Barnett, and A. M. Yao, Chirality and the angular momentum of light, Phil. Trans. R. Soc. A 375, 20150433 (2017).

[41] F. Alpeggiani, K. Y. Bliokh, F. Nori, and L. Kuipers, Electromagnetic Helicity in Complex Media, Phys. Rev. Lett. 120, 243605 (2018).

[42] D. L. Andrews, Quantum formulation for nanoscale optical and material chirality: Symmetry issues, space and time parity, and observables, J. Opt. 20, 033003 (2018).

[43] I. Myrgorodska, C. Meinert, S. V. Hoffmann, N. C. Jones, L. Nahon, and U.J. Meierhenrich, Light on chirality: Absolute asymmetric formation of chiral molecules relevant in prebiotic evolution, ChemPlusChem 82, 74 (2017).

[44] P. Lodahl, S. Mahmoodian, S. Stobbe, A. Rauschenbeutel, P. Schneeweiss, J. Volz, H. Pichler, and P. Zoller, Chiral quantum optics, Nature (London) 541, 473 (2017).

[45] M. Schäferling, Chiral Nanophotonics (Springer International Publishing, Switzerland, 2017).

[46] D. Chelouche and E. I. Guendelman, Cosmic analogs of the Stern-Gerlach experiment and the detection of light bosons, Astrophys. J. 699, L5 (2009). 


\title{
SUPPLEMENTARY INFORMATION \\ A chiral optical Stern-Gerlach Newtonian experiment
}

\author{
Nina Kravets, Artur Aleksanyan, and Etienne Brasselet*
}

November 6, 2018

Univ. Bordeaux, CNRS, Laboratoire Ondes et Matière d'Aquitaine, F-33400 Talence, France.

${ }^{*}$ Corresponding author: etienne.brasselet@u-bordeaux.fr

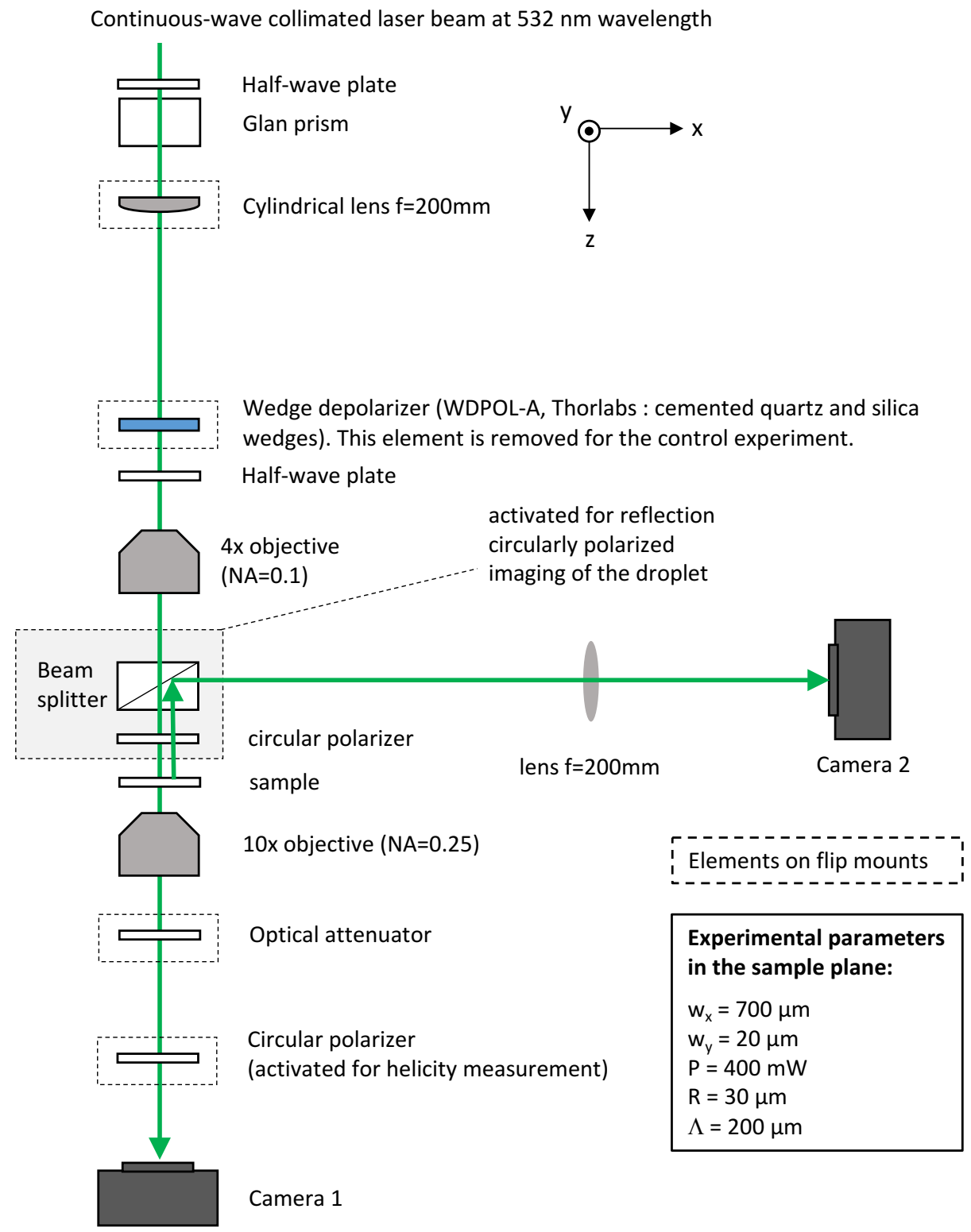

Supplementary Fig. 1 Details of the full experimental setup. 